\title{
ARA55 in Cancers: A Double-Edged Sword
}

\author{
Zhaolei Cui* and Yan Chen* \\ Department of Clinical Laboratory, Fujian Provincial Key Laboratory of Tumor Biotherapy, Fujian Cancer Hospital \& \\ Fujian Medical University Cancer Hospital, Fuzhou, Fujian, RP China
}

*Corresponding author: Zhaolei Cui and Yan Chen, Department of Clinical Laboratory, Fujian Provincial Key Laboratory of Tumor Biotherapy, Fujian Cancer Hospital \& Fujian Medical University Cancer Hospital. No.420 Fuma Road, Jin'an District, Fuzhou 350014, PR China

\begin{tabular}{|c|c|}
\hline ARTICLE INFO & ABSTRACT \\
\hline Received: 幽 February 05, 2020 & Androgen receptor coactivator $55 \mathrm{kDa}$ protein (ARA55) was initially isolated \\
\hline Published: March 04, 2020 & $\begin{array}{l}\text { and identified from mouse MC3T3-E1 osteoblasts treated with transforming growth } \\
\text { factor- } \beta 1 \text { (TGF } \beta 1 \text { ). So, it is also named as TGF } \beta 1 \text {-induced transcript } 1 \text { protein (TGF } \beta 1 \text { II). }\end{array}$ \\
\hline $\begin{array}{l}\text { Citation: Zhaolei Cui, Yan Chen. ARA55 in } \\
\text { Cancers: A Double-Edged Sword. Biomed J Sci } \\
\text { \& Tech Res 26(2)-2020. BJSTR. MS.ID.004326. }\end{array}$ & $\begin{array}{l}\text { Thus far, the gene or amino acid sequence, structure, biological properties as well as } \\
\text { peptide synthesis of ARA55 have been well studied. Current studies suggest that ARA55 } \\
\text { functions as a molecular adapter in coordinating multiple Protein-Protein Interactions } \\
\text { (PPIs) in Focal Adhesion (FA) complexes and the nucleus. Besides, accumulating } \\
\text { evidence has shown that ARA55 is also implicated in tumorigenesis and the progression } \\
\text { of cancers. However, there are a lot of disputes on the functional role of ARA55 in } \\
\text { different cancers. This review summarized the recent findings on ARA55 with particular } \\
\text { emphasis on its role in tumors. }\end{array}$ \\
\hline
\end{tabular}

Abbreviations: ARA55: Androgen Receptor Coactivator 55kDa protein, AR: Androgen Receptor, CRIP2: Cysteine-Rich Protein 2, EMT: Epithelial-To-Mesenchymal Transitions, FA: Focal Adhesion, Hic-5: Hydrogen Peroxide-Inducible Clone 5 protein, MAPK15: MitogenActivated Protein Kinase 15, ORF: Open Reading Frame, PCCs: Pancreatic Cancer Cells, PPIs: Protein-Protein Interactions, PTPN12: Protein Tyrosine Phosphatase Non-Receptor Type 12, SMAD3: Smad Family Member 3, TGF $\beta 1$ : Transforming Growth Factor- $\beta 1$.

\section{Introduction}

ARA55, belonging to the LIM protein family and known as TGF $\beta 11$ or hydrogen peroxide-inducible clone 5 protein (Hic-5), is initially isolated and identified from mouse MC3T3 osteoblasts induced by TGF $\beta 1$ and $\mathrm{H}_{2} \mathrm{O}_{2}$ [1]. Human ARA55 gene is located at chr16: 31,471,585-31,477,960 (GRCh38/hg38) and contains 11 exons. With an Open Reading Frame (ORF) of 1386bp in length, it can encode a protein polypeptide composed of 461 amino acids with a molecular weight of $55 \mathrm{kDa}$. Allowing for the similar $4 \mathrm{LD}$ domains in the $\mathrm{N}$-terminal region and 4 LIM zinc finger domains in the C-terminal segment along with 55\% amino acid sequence consistent with that of paxillin, and the molecular structure of ARA55 has 72\% similarity to paxillin [2]. Specifically, the LIM zincbinding domains play important roles in mediating glucocorticoid receptor coactivation and interacting with multiple proteins such as Androgen Receptor (AR), Cysteine-Rich Protein 2 (CRIP2) and Smad Family Member 3 (SMAD3). The second and third LIM zinc- binding domains are involved in mediating expressions of FAs and actin stress fibers, and the third and fourth LIM zinc-binding domains in the interaction with protein tyrosine Phosphatase NonReceptor Type 12 (PTPN12) and mitogen-activated protein kinase 15 (MAPK15).

The fourth LIM zinc-binding singly interacts with homooligomerization and targets to the nuclear matrix. Therefore, the unique protein structure endows distinctive biofunctions of ARA55. Recently, it has been found that ARA55 as a coactivator interacts with multiple targets and enhance the transcriptional activity of AR--a transcription factor which is activated by androgen and essential in male sexual differentiation $[1,2]$. Despite the role as a molecular adapter coordinating multiple PPIs in FA complexes and the nucleus, it is also involved in the cell growth, proliferation, migration, differentiation and senescence particularly in cancers [2]. In this review, we have summarized the distribution of ARA55 
in tissues and its role in Epithelial-To-Mesenchymal Transitions (EMT) and given high priority to its biofunctions in cancers.

\section{The Low Expression of Ara55 In Somatic Tissues and Cell Lines}

ARA55 has a low tissue specificity with a low-to-medium expression in the cytoplasm in most tissues including smooth muscle (Figure 1). ARA55 also reveals a low cell line specificity. The immunofluorescence staining shows that ARA55 protein is mainly localized to FA sites and the cytosol in both U-2 OS and U-251MG cell lines, and to the cytosol of A-431 cells (Figure 2A). The RNA cell line category shows that cell lines derived from brain, skin, breast, sarcoma, fibroblast, endothelial and miscellaneous retain a medium level of ARA55 RNA (Figure 2B).

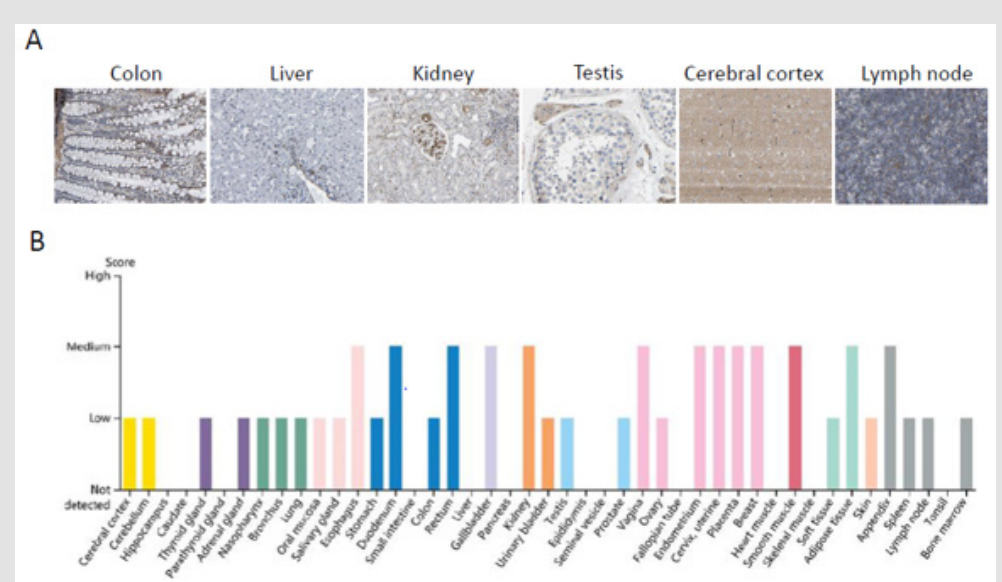

Figure 1: Immunohistochemistry assay reveals that ARA55 has low tissue specificity in most tissues.

A. The expression and distribution of ARA55 in colon, liver, kidney, testis, cerebral cortex, and lymph node.

B. The expression of ARA55 protein in normal tissues. Note: Data were downloaded from the HUMAN PROTEIN ATLAS.

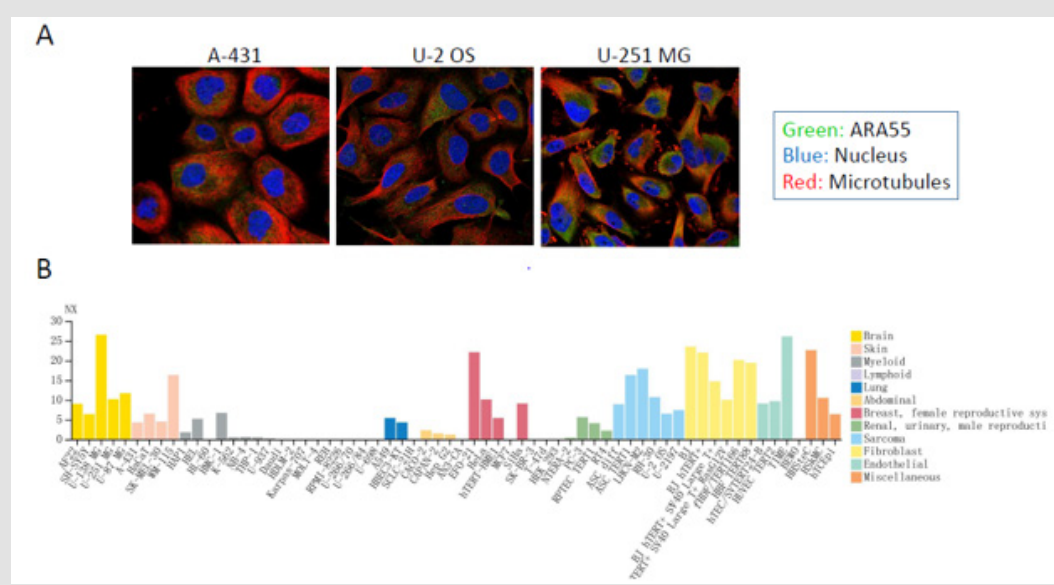

Figure 2: The expression and location of ARA55 in normal tissue cell lines by immunofluorescence staining.

A. The cellular location of human ARA55 in A-431, U-2 OS, and U-251MG cell lines.

B. The expression of ARA55 RNA in normal tissue cell lines. Note: Data were downloaded from the HUMAN PROTEIN ATLAS

\section{The Involvement of Ara55 In Emt}

As ARA55 mainly exists in FAs and nuclei [3-6], it is found to shuttle between the cytoplasm and nucleus and participate in several important physiological processes such as cell adhesion, EMT, cell growth and migration induced by TGF $\beta 1$ [7-15]. In the nucleus, ARA55 regulates the transcription activity of corticosteroids, androgens, mineralocorticoids and progesterone receptors as a receptor coactivator through phosphorylation or mediating PPIs [4-6]. Combining with Hsp27, it is also involved in the TGF $\beta$-induced adhesion of vascular smooth muscle cells [14]. In normal breast epithelial MCF10A cells, the phosphorylation level of ARA55 protein significantly increases with the stimulation of TGF $\beta$ [13]. Meanwhile, TGF $\beta$-induced high levels of ARA55 facilitate the degradation of matrix in MCF10A cells and the formation of dendrite-like invadopodia to generate EMT a key link in the physiological process of cell invasion and migration. So ARA55 plays an important role in EMT induced by TGF 1 [10-13]. 
The Pathological Significance of Varied Ara55 Levels in Cancers

ARA55 is up regulated in several types of cancers compared with normal tissues. In cancer tissues, the expression peaks of ARA55 RNA are higher in several cases of prostate, pancreatic, colorectal, breast, liver and lung carcinomas, etc. (Figure 3). Currently, a handful of studies have investigated biofunctions of ARA55 in tumors, however, the results are controversial. Gulvady et al. have found that ARA55 affects the morphological changes and plasticity of cancer cells during invasion and metastasis [15]. Wu et al. have studied its ultrastructure by electron microscope and found that accompanied by the up-regulated expression of ARA55 the transplantation tumor tissue cells and LoVo colorectal cancer cells develop well and tend to mature with an increased number of intercellular connections, indicating that ARA55 may participate in the occurrence and development of colorectal cancer [16]. ARA55 serves as an activator of steroid hormone receptor in the occurrence of prostate cancer, showing the potential to promote tumor metastasis [17-19]. In breast cancer, ARA55 has been found to be involved in the regulation of tumor adhesion, invasion and migration [20].

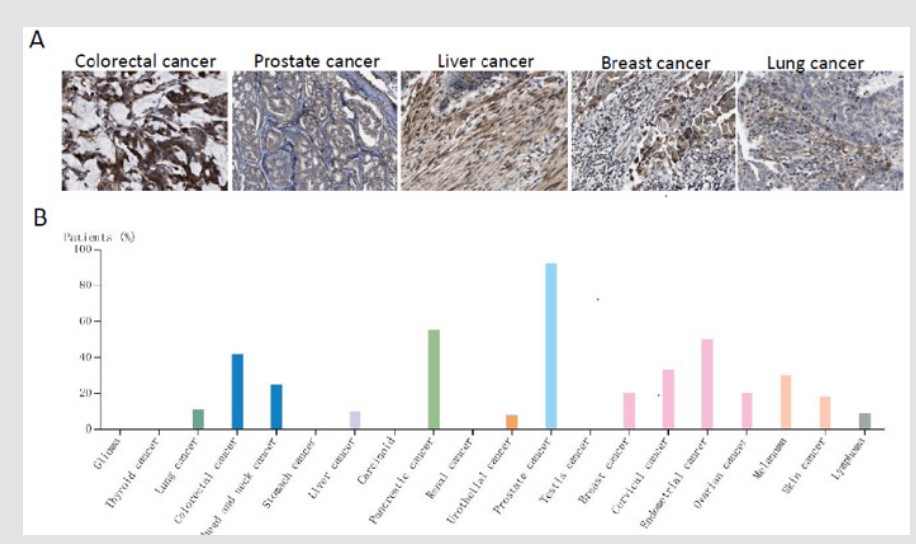

Figure 3: The expression and location of ARA55 in cancers.

A. Moderate granular cytoplasmic staining is adopted in colorectal, prostate, breast, liver, and lung carcinomas and the moderate granular cytoplasmic staining is confirmed.

B. Several cases of prostate colorectal, breast, endometrial, pancreatic cancers and squamous cell carcinomas reveals the moderate granular cytoplasmic staining by immunohistochemistry, and the remaining cancers are weakly stained or negative.

Note: Data were downloaded from the HUMAN PROTEIN ATLAS.
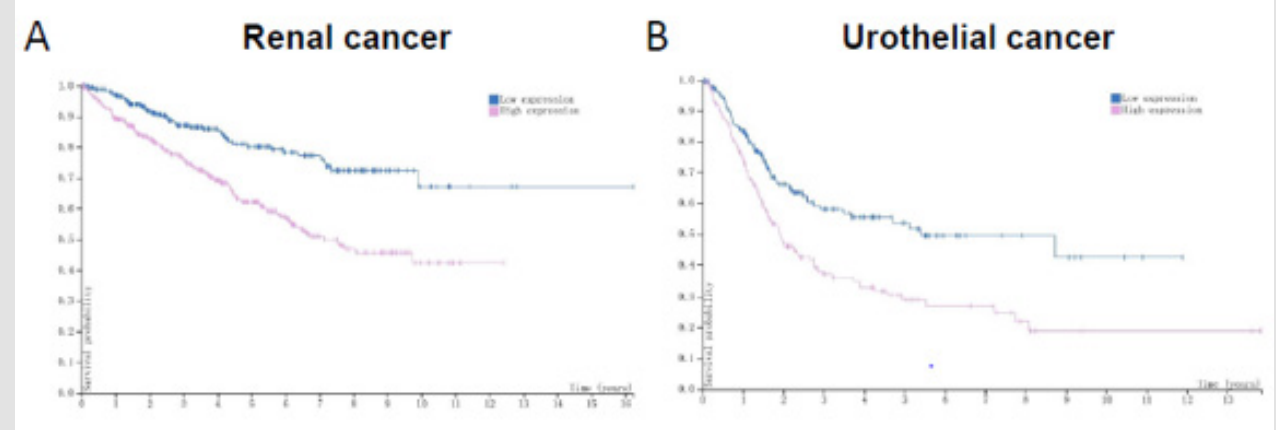

Figure 4: ARA55 servers as a prognostic marker in cancers.
A. Renal cancer
B. Urothelial cancer. Note: Data were downloaded from the HUMAN PROTEIN ATLAS.

Besides, ARA55 as a scaffold protein contributes to the formation of abdominal aortic aneurysm through MKK4 / p54 / JNK signaling pathway [21]. Qian, et al. [22] have found that ARA55 is highly expressed in pancreatic cancer, and the higher the expression level of ARA55 is, the worse the prognosis of patients will be. The downregulation of ARA55 in pancreatic cancer cells (PCCs) significantly inhibits cell proliferation, invasion and metastasis, and induces tumor cell apoptosis. Noguchi, et al. [23] 
confirm that there is a high expression of ARA55 in B16-F1 mouse melanoma cells. When the expression of ARA55 has been silenced by shRNA, the cell growth, proliferation, invasion and migration are inhibited to different degrees. In Hepatocellular Carcinoma (HCC), the expression of ARA55 is closely associated with tumor progression [24]. Data from the HUMAN PROTEIN ATLAS reveal that ARA55 can be rated as a prognostic biomarker in renal cancer and urothelial cancer (Figure 4), as those cancer patients with high protein levels of ARA55 have yielded unfavourable prognosis. Therefore, it can be inferred that ARA55 acts as an oncogene in these tumors. Nevertheless, others suggest that ARA55 acts as a tumor suppressor gene.

Evidences show that the expression level of ARA55 in colorectal cancer is significantly lower than that in adjacent tissues; and the overexpression of ARA55 in colorectal cancer LoVo cells triggers endogenous mitochondrial apoptosis [25]. In prostate cancer, the expression of ARA55 significantly decreases and is found to be negatively correlated with the degree of tumor differentiation. The silenced expression of ARA55 has also been found in some prostate cancer cell lines $[17,18]$. In our previous research, the overexpression of ARA55 has inhibited the growth, proliferation, invasion and metastasis of CNE2 nasopharyngeal carcinoma cells in vitro and has ultimately triggered cell apoptosis. Apart from that, exogenous-ARA55-induced Bcl-2 down-regulation stimulates a series of cascade reactions: a significantly increased expression of downstream cytochrome c followed by the largely unleashed spliceosome of caspase- 9 along with an activation of caspase- 3 (unpublished data).

\section{Summary}

ARA55 as a multi-domain protein can bind to a variety of structural proteins and signal proteins, which means it is involved in the formation of FAs in extracellular matrix and bridges the upstream and downstream proteins in a signal pathway. It also plays as a co-promoter of nuclear receptors in the activation of target genes. Like a double-edged sword, the biofunction of ARA55 in malignant tumors varies according to the tumor type. Advancing research on the biofunctions and mechanisms of ARA55 in malignant tumors will unveil the key link in this variation.

\section{Acknowledgement}

This study was supported by Provincial Natural Science Fund of Fujian (Grant no. 2017J05120) and Science and Technology Program of Fujian Province, China (Grant no. 2018Y2003).

\section{Conflicts of Interest}

The authors declare no conflict of interest in any form.

\section{References}

1. Shibanuma M, Mashimo J, Kuroki T, Nose K (1994) Characterization of the TGF beta 1-inducible hic- 5 gene that encodes a putative novel zinc finger protein and its possible involvement in cellular senescence. J Biol Chem 269(43): 26767-26774
2. Turner CE (2000) Paxillin and focal adhesion signalling. Nat Cell Biol 2(12): E231-236

3. Shola DT, Wang H, Wahdan-Alaswad R, Danielpour D (2012) Hic-5 controls BMP4 responses in prostate cancer cells through interacting with Smads 1, 5 and 8. Oncogene 31(19): 2480-2490.

4. Shibanuma M, Kim Kaneyama JR, Sato S, Nose K (2004) LIM protein, Hic-5, functions as a potential coactivator for Sp1. J Cell Biochem 91(3): 633-645.

5. Li Bo (2013) Research progression of Hic-5/ARA55protein. Int J Lab Med 34(1): 65-68.

6. Heitzer MD, DeFranco DB (2007) Hic-5/ARA55: A prostate stromaspecific AR coactivator. Steroids 72(2): 218-220.

7. Fernandez I, Martin-Garrido A, Zhou DW, Clempus RE, Seidel-Rogol B, et al. (2015) Hic-5 Mediates TGFß-Induced Adhesion in Vascular Smooth Muscle Cells by a Nox4-Dependent Mechanism. Arterioscler Thromb Vasc Biol 35(5): 1198-1206.

8. Dabiri G, Tumbarello DA, Turner CE, Van de Water L (2008) TGF-beta1 slows the growth of pathogenic myofibroblasts through a mechanism requiring the focal adhesion protein, Hic-5. J Invest Dermatol 128(2): 280-291.

9. Jamba A, Kondo S, Urushihara M, Nagai T, Kim-Kaneyama JR (2015) Hydrogen peroxide-inducible clone-5 regulates mesangial cell proliferation in proliferative glomerulonephritis in mice. PLoS One 10(4): e0122773.

10. Tumbarello DA, Turner CE (2007) Hic-5 contributes to epithelialmesenchymal transformation through a RhoA/ROCK-dependent pathway. Journal of cellular physiology 211(3): 736-747.

11. Deakin NO, Pignatelli J, Turner CE (2012) Diverse roles for the paxillin family of proteins in cancer. Genes Cancer 3(5-6): 362-370.

12. Derynck R, Muthusamy BP, Saeteurn KY (2014) Signaling pathway cooperation in TGF- $\beta$-induced epithelial-mesenchymal transition. Curr Opin Cell Biol 31: 56-66.

13. Pignatelli J, Tumbarello DA, Schmidt RP, Turner CE (2012) Hic-5 promotes invadopodia formation and invasion during TGF- $\beta$-induced epithelial-mesenchymal transition. J Cell Biol 197(3): 421-437.

14. Fernandez I, Martin-Garrido A, Zhou DW, Clempus RE, Seidel-Rogol B, et al. (2015) Hic-5 Mediates TGFß-Induced Adhesion in Vascular Smooth Muscle Cells by a Nox4-Dependent Mechanism. Arterioscler Thromb Vasc Biol 35(5): 1198-1206.

15. Gulvady AC, Dubois F, Deakin NO, Goreczny GJ, Turner CE (2018) Hic5 expression is a major indicator of cancer cell morphology, migration, and plasticity in three-dimensional matrices. Mol Biol Cell 29(13): 17041717.

16. Wu Yingchao, Wang Xin, Liu Yucun, Wan Yuanlain, Zhu Jing (2010) Effects of hydrogen peroxide-inducible clone 5/androgen receptorassociated protein 55 on the ultrastructure of the human colorectal cancer cells and subcutaneously implanting tumors. Chinese Journal OF Experimental Surgery 27(8): 1027-1028.

17. Yang Nianqin, Li Runsheng, Li Dong, Sha Jianjun, Li Ping, et al. (2007) Expression of ARA55 mRNA in Prostate Carcinoma Tissues and Its Significance. National Journal of Andrology 13(8): 696-699.

18. Li Zeng, Wang Delin, Lan Jianhua, Yang Zongke (2009) Expression of Hic-5/ARA55 in human prostate tissues and its clinical significance. Guangdong Medical Journal 30(10): 1526-1528.

19. Shibanuma M, Mori K, Nose K (2012) HIC-5: A Mobile Molecular Scaffold Regulating the Anchorage Dependence of Cell Growth. Int J Cell Biol 2012: 426138.

20. Deakin NO, Turner CE (2011) Distinct roles for paxillin and Hic-5 in regulating breast cancer cell morphology, invasion, and metastasis. Mol Biol Cell 22(3): 327-341. 
21. Lei XF, Kim-Kaneyama JR, Arita-Okubo S, Offermanns S, Itabe H, et al. (2014) Identification of Hic-5 as a novel scaffold for the MKK4/p54 JNK pathway in the development of abdominal aortic aneurysms. J Am Heart Assoc 3(3): e000747.

22. Qian B, Wei L, Yang Z, He Q, Chen H, et al. (2020) Hic-5 in pancreatic stellate cells affects proliferation, apoptosis, migration, invasion of pancreatic cancer cells and postoperative survival time of pancreatic cancer. Biomed Pharmacother 121: 109355.

23. Noguchi F, Inui S, Nakajima T, Itami S (2012) Hic-5 affects proliferation, migration and invasion of B16 murine melanoma cells. Pigment Cell Melanoma Res 25(6): 773-782.

\section{ISSN: 2574-1241}

DOI: $10.26717 /$ BJSTR.2020.26.004326

Zhaolei Cui. Biomed J Sci \& Tech Res

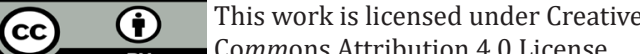

Submission Link: https://biomedres.us/submit-manuscript.php
24. Wu JR, Hu CT, You RI, Pan SM, Cheng CC, et al. (2015) Hydrogen peroxide inducible clone-5 mediates reactive oxygen species signaling for hepatocellular carcinoma progression. Oncotarget 6(32): 32526-32544.

25. Cui Wei, Wang Xin, Liu Yucun, Wan Yuanlian, Guo Hongjie, et al. (2006) Expression of HIC-5/ARA55 in colonrectal cancer and its mechanisms of action. Journal of Pejing University (Health Sciences) 38(3): 280-283.

BIOMEDICAL
RESEARCHES $\quad$\begin{tabular}{l} 
Assets of Publishing with us \\
\hline
\end{tabular}

